\title{
Simultaneous Integrated Model with Multiobjective for Continuous Berth Allocation and Quay Crane Scheduling Problem
}

\author{
${ }^{1}$ Department of Mathematical Sciences, Faculty of Science, \\ Universiti Teknologi Malaysia, Johor, Malaysia \\ ${ }^{2}$ Department of Mathematical Sciences, Faculty of Science \\ and Universiti Teknologi Malaysia - Centre of Industrial \\ and Applied Mathematics, Universiti Teknologi Malaysia, \\ Johor, Malaysia \\ E-mail:nurhidayu5@live.utm.my; zmarlizawati@utm.my
}

Nurhidayu Idris $^{1}$ and Zaitul Marlizawati Zainuddin ${ }^{2}$

Received 23 October 2016; Accepted 24 November 2016;

Publication 19 December 2016

\begin{abstract}
This paper presents the simultaneous integration model of berth allocation and quay crane scheduling. Berths and quay cranes are both critical resources in port container terminals. The mathematical model uses a mixed integer linear programming with multiple objectives generated by considering various practical constraints. Small data instances have been taken to validate the integrated model. A numerical experiment was conducted by using LINGO programming software to evaluate the performance and to obtain the exact solution of the suggested model.
\end{abstract}

Keywords: Berth allocation, Quay crane scheduling, Multi objectives, Integration.

Journal of Industrial Engineering and Management Science, Vol. 1, 1-12.

doi: 10.13052/jiems2446-1822.2016.006

(C) 2016 River Publishers. All rights reserved. 


\section{Introduction}

In today's global economy, shipping, port and logistics activities are well-known and indispensable. They have an important role to play in facilitating the global trade nowadays. As we can observe, most of the businesses around the world involve shipping from one destination to other destination. Since the water transportation becomes an essential need for human daily activities especially in business field, it has caused a great demand for port container terminals. A great demand for seaport container terminals recently in business, there exist the systematic operation competition between ports. Regarding to the fierce competition among container terminals, high performance with an efficient processing in a container terminal is vital as mentioned in [1]. Previously, Daganzo [2] and Park and Kim [3] were both first presented the integrated approaches. Both papers highlighted applying monolithic model for crane assignment and crane scheduling as in [2] and berth allocation with crane assignment as in [3]. Generally, the handling time of the ship is affected by the crane schedule. The integration concept of Berth Allocation Problem (BAP) and Quay Crane Scheduling Problem (QCSP) was proposed by Park and Kim [3] with continuous berth model. The problem is formulated using the mixed integer programming (MIP) model whereby both time and space were discretized and a two phased procedure was designed to solve the problem. In the first phase, berth allocation and rough quay crane allocation was determined while in the second phase by using the solution found from the first phase, a detailed individual crane scheduling was generated. Next, this study has been extended as in [4] by restricting the moving range of each crane individually at the quay. The differences exist between these two papers are they allocate the two resources which are berths and quay cranes simultaneously, not sequentially like in [3].

In addition, the simultaneous concept has been studied as in [5] on the integration of berth allocation and quay crane scheduling problem. This problem was designed simultaneously between scheduling of cranes for all vessels and assigning of vessels to the berths. Continuous layout of berth was chosen in order to be more practical and less time consumed by vessels at port. Aykagan [5] proposed to solve the integration problem by applying a Tabu search heuristic with the objectives to minimize the serving time and weighted tardiness of vessels. Besides that, the same simultaneous concept was proposed in [6] for the integration model of BAP and QCSP. The integrated optimization model on berth allocation and quay crane scheduling was built with the objective to reduce the operating costs of quay crane and vessels. 
Wang, Cao, Wang and $\mathrm{Li}$ [6] as well developed a new genetic and ant colony algorithm whereby the partial allocation plan is solved using genetic algorithm, then adjusted against berth through ant colony algorithm to find an optimal solution. Compared to Aykagan [5], Wang, Cao, Wang and Li [6] focused on a discrete berth layout rather than continuous berth. However, both papers focused on a dynamic concept for the arrival of ships. The integration of BAP and QCSP also has been studied as in [1] on a simultaneous process. The study is solved in a simultaneous way with uncertainties on arrival time of vessel and container handling time. The spatial and temporal constraint applied in [1] is similar to [6] whereby discrete layout of berths with dynamic arrival of vessels. A simulation based on Genetic Algorithm (GA) search procedure is built in order to generate robust berth and QC schedule proactively.

\section{Problem Description}

Mainly, the focus in this research is to develop a simultaneous Integration Continuous Berth Allocation Problem and Quay Crane Scheduling Problem (IBAPCQCSP) while considering multiple objectives. This paper involves two processes namely, berth allocation and quay crane scheduling which are vital operations in container terminal. Thus, the flow of the operations has been presented in the mathematical model throughout this paper. The handling time of vessel depends on the resources utilization efficiency which may result an early departure of vessel. This research focuses on continuous berth layout whereby the vessels can be served wherever empty spaces are available along the quay. Next, to formulate the multi objectives model of simultaneous IBAPCQCSP, below assumptions need to be followed:

1. The length of each vessel represents through number of holds of 2,3 or 4 holds whereby only one crane can work on a hold at one time.

2. Numbers of vessels can berth along the quay and being served immediately.

3. During the planning horizon, vessels can arrive at port and cannot be served before its arrival.

4. Once all holds on a vessel is processed by quay cranes, a vessel is completed.

5. Once the cranes start to work on a hold, the work has to be continued until the hold completely being served.

6. Quay cranes cannot cross each other while on the same tracks. 
7. After the process of loading and unloading container is completed on all holds, only then a vessel can leave the port.

8. Handling time of vessel depends on the number of quay cranes assigned to the vessel.

9. Quay cranes can move from hold to hold either in the same or different vessel without crossing one another.

\section{Mathematical Formulation}

In this section, a multiple objectives mathematical model is formulated with the simultaneous integrated berth allocation and quay crane scheduling. The study focuses on the dynamic arrival of vessels where a set $V$ of vessels with known arrival times, whereby $n=|V|$. For each vessel $i \in V$, the parameter and formulation applied in this study is defines as below:

$L$ : set of berths along the quay

$Q$ : a set of non-identical quay cranes working on a single set of rails.

$n_{q}$ : travel time of quay crane $q$,

$T$ : time period of vessels

$V:$ a set of vessels

$M$ : a large scalar number

$h_{i}$ : number of holds of vessel $i$,

$\mathrm{pr}_{i}^{k}$ : processing time of hold $k$ of vessel $i$,

$p r_{i}^{\max }$ : maximum hold processing time for vessel $i$

$p r_{i}^{\max }: \max _{i}\left\{p r_{i}^{k}\right\}$

$f_{i}$ : lateness penalty time of vessel $i$,

$d_{i}$ : due time of vessel $i$ (where $d_{i}, a_{i}+p r_{i}^{*}$ )

$a_{i}$ : arrival time of vessel $i$,

$b p_{i}$ : berthing position of vessel $i$,

$b t_{i}$ : berthing time of vessel $i$,

$e_{i}$ : the earliest time that vessel $i$ can depart.

$x_{i j}= \begin{cases}1 & \text { if vessel } i \text { berth after vessel } j \text { departs, } \\ 0 & \text { otherwise }\end{cases}$

$y_{i j}= \begin{cases}1 & \text { if vessel } i \text { berth completely above vessel } j \text { on the } \\ & \text { time-space diagram, } \\ 0 \quad & \text { otherwise }\end{cases}$ 


$$
\begin{aligned}
r_{i q k}^{t} & = \begin{cases}1 & \text { if at least one quay crane is assigned on hold } k \text { of vessel } \\
0 & i \text { at time } t\end{cases} \\
w_{i}^{t} & = \begin{cases}1 & \text { otherwise; } \\
0 & \text { otherwise; }\end{cases}
\end{aligned}
$$

The simultaneous concept of IBAPCQCSP model with multiple objectives and dynamic arrival of vessels is formulated as follow:

$$
\operatorname{Min} \sum_{i=1}^{n}\left(e_{i}-a_{i}\right)+\sum_{i=1}^{n} f_{i}\left(e_{i}-d_{i}\right)+\sum_{i=1}^{n} \sum_{t \in T} \sum_{q \in Q} \sum_{k=1}^{h_{i}} n_{q} r_{i q k}^{t}
$$

subject to

$$
\begin{aligned}
x_{i j}+x_{j i}+y_{i j}+y_{j i} \geq 1 & \forall i, j \in V \text { and } i<j \\
x_{i j}+x_{j i} \leq 1 & \forall i, j \in V \text { and } i<j \\
y_{i j}+y_{j i} \leq 1 & \forall i, j \in V \text { and } i<j \\
b t_{j} \geq e_{i}+\left(x_{i j}-1\right) M & \forall i, j \in V \text { and } i<j \\
b p_{j} \geq b p_{i}+h_{i}+\left(y_{i j}-1\right) M & \forall i, j \in V \text { and } i<j \\
b t_{i} \geq a_{i} & \forall i \in V \\
b t_{i} \geq t r_{i q k}^{t}+\left(1-r_{i q k}^{t}\right) T & \forall i \in V, \forall k \in\left\{1, \ldots, h_{i}\right\}, \\
e_{i} \geq t r_{i q k}^{t}+p r_{i}^{k} & \forall i \in V, \forall k \in\left\{1, \ldots, h_{i}\right\}, \\
e_{i} \geq b t_{i}+p r_{i}^{\max } & \forall i \in V \\
w_{i}^{\min } \leq \sum_{i \in V} r_{i q k}^{t} \leq w_{i}^{\max } & \forall q \in Q, \forall k \in\left\{1, \ldots, h_{i}\right\}, \\
\sum_{i \in V} \sum_{q \in Q} \sum_{k=1}^{h_{i}} r_{i q k}^{t} \leq Q & \forall t \in T
\end{aligned}
$$




$$
\begin{array}{cl}
\sum_{q \in Q} r_{i q k}^{t}=w_{i}^{t} & \forall i \in V, \forall k \in\left\{1, \ldots, h_{i}\right\}, \\
& \forall t \in T \\
\sum_{t \in T} w_{i}^{t}=e_{i}-b t_{i} & \forall i \in V \\
1 \leq b p_{i} \leq L-h_{i}+1 \quad & \forall i \in V \\
x_{i j} \in\{0,1\}, y_{i j} \in\{0,1\} & \forall i, j \in V \text { and } i \neq j \\
r_{i q k}^{t} \in\{0,1\}, w_{i}^{t} \in\{0,1\} & \forall i \in V, \forall k \in\left\{1, \ldots, h_{i}\right\}, \\
& \forall t \in T, \forall q \in Q
\end{array}
$$

Referring to mathematical formulation above, the first objective that needs to be achieved is to minimize the duration of vessels at port and second objective is to reduce the waiting time of vessels to be served. The last objective is to lessen the travelling time of quay cranes. Constraints (2) through (4) are applied to prevent the overlapping rectangle of vessels. Constraints (5) and (6) ensure that the chosen berthing times and berthing positions are consistent with the definitions of $x_{i j}$ and $y_{i j}$ with $M$ as a large positive number. Constraint (7) forces the berthing of vessels must be after their arrivals and constraint (8) forces the work on hold of vessels to begin immediately after berthing. Constraint (9) ensures that vessel can leave the port after all holds finished processing by quay cranes. Constraint (10) is to provide the lower bound on the completion time of vessel, $e_{i}$ given berthing time, $b t_{i}$. Constraint (11) ensures that only one to four quay cranes can serve a vessel at the same time. Constraint (12) ensures that the working quay cranes at any time period cannot exceeded $Q$, and constraint (13) ensures the consistency between $w_{i}^{t}$ and $r_{i q k}^{t}$ whereby the number of quay cranes operated on vessel $i$ at time $t$ are equal to the summation of quay cranes assigned to the holds of vessel $i$ at time $t$. Constraint (14) sets the number of quay cranes work for the vessels must be equal to the duration time of vessels being served. Constraint (15) is to make sure that the vessels fit on berths along the quay.

\subsection{Simple Numerical Example}

The model formulation presented previously was validated by using commercial programming software, LINGO 15.0 for small problem which can be seen in Table 1 with the length of berth, $L=7$ and quay cranes, $Q=4$. 
Table 1 A small instance for simultaneous IBAPCQCSP

\begin{tabular}{lllllc}
\hline$i$ & 1 & 2 & 3 & 4 & 5 \\
\hline$a_{i}$ & 2 & 1 & 3 & 1 & 4 \\
$d_{i}$ & 8 & 4 & 9 & 5 & 11 \\
$f_{i}$ & 3 & 4 & 3 & 3 & 4 \\
$h_{i}$ & 2 & 3 & 3 & 4 & 4 \\
$p r_{i}^{1}$ & 3 & 2 & 2 & 3 & 3 \\
$p r_{i}^{2}$ & 4 & 2 & 4 & 1 & 2 \\
$p r_{i}^{3}$ & - & 2 & 1 & 0 & 2 \\
$p r_{i}^{4}$ & - & - & - & 1 & 4 \\
\hline
\end{tabular}

Table 2 A travel time of quay cranes (hours)

\begin{tabular}{ccccc}
\hline$q$ & 1 & 2 & 3 & 4 \\
\hline$n_{q}$ & 0.10 & 0.20 & 0.15 & 0.25 \\
\hline
\end{tabular}

In this study, the length of vessel is represented through the different number of holds for each vessel which are 2, 3 or 4 holds. The vessels can moor at the berth along the quay and obtain service by quay cranes simultaneously with only one crane can assign and works on a hold. The processing time of each hold has been assigned for every vessel to be served by quay cranes. The quay crane is allowed to shift form one hold of a vessel to another only if a quay crane finished processing initially assigned hold. Each vessel is considered completed once the quay cranes finished processing all holds in a vessel. After all the vessels are finished servicing, the exact solution is obtained and presented through a time-space diagram or Gantt chart which is the best way to represent a vessel who is queuing to be served and in the middle of servicing.

\subsection{Main Results}

From the simple numerical data assigned, the exact solutions by using three different rules are compared. Table 3 and Figure 1 shows the exact solution of using First Come First Serve (FCFS) rule while Table 4 and Figure 2 shows the result obtained by applying Large Vessel First (LVF) rule. For the last result, Shortest Processing Time First (SPTF) rule is applied and the exact solution can be seen in Table 5 and Figure 3 as followed. The moving path of the quay cranes also will be presented in the time space diagram.

Based on the three exact solutions diagrams above, the total objective function value obtained by applying FCFS rule was 40.4 hours with the idle cranes of 8 while LVF rule with total optimal objective function value of 
Table 3 The exact solution for simultaneous IBAPCQCSP (FCFS rule)

\begin{tabular}{lllccc}
\hline$i$ & 1 & 2 & 3 & 4 & 5 \\
\hline$b p_{i}$ & 1 & 1 & 5 & 4 & 1 \\
$b t_{i}$ & 3 & 1 & 6 & 1 & 7 \\
$e_{i}$ & 7 & 3 & 11 & 6 & 12 \\
$r_{i q}^{1}$ & 3 & 1 & 6 & 1 & 7 \\
$r_{i q}^{2}$ & 3 & 1 & 6 & 4 & 7 \\
$r_{i q}^{3}$ & - & 1 & 10 & - & 9 \\
$r_{i q}^{4}$ & - & - & - & 5 & 8 \\
\hline
\end{tabular}

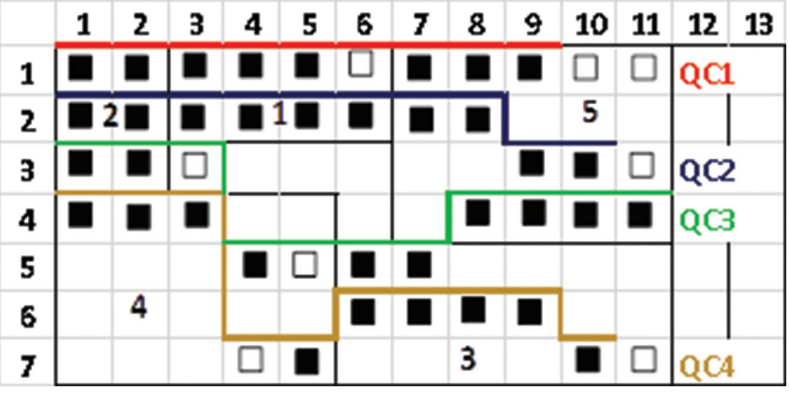

Figure 1 A time space diagram for the exact solution of simultaneous IBAPCQCSP (FCFS rule).

Table 4 The exact solution for simultaneous IBAPCQCSP (LVF rule)

\begin{tabular}{lccccc}
\hline$i$ & 1 & 2 & 3 & 4 & 5 \\
\hline$b p_{i}$ & 1 & 5 & 5 & 1 & 1 \\
$b t_{i}$ & 8 & 2 & 4 & 1 & 4 \\
$e_{i}$ & 12 & 4 & 10 & 4 & 8 \\
$r_{i q}^{1}$ & 8 & 2 & 8 & 1 & 4 \\
$r_{i q}^{2}$ & 8 & 2 & 4 & 1 & 4 \\
$r_{i q}^{3}$ & - & 2 & 8 & - & 6 \\
$r_{i q}^{4}$ & - & - & - & 1 & 4 \\
\hline
\end{tabular}

33.2 hours and 8 idle cranes. For SPTF rule, the total objective function value obtained was 28.35 hours with 8 idle cranes too. It shows that the performance of the integrated operation regarding BAP and QCSP by using the SPTF rule is smaller compared to FCFS and LVF rules. Therefore, the SPTF rule is more applicable and effective for the model to be used in further research. From the results, SPTF rule gives the lowest results since the berth was fully occupied with vessels from time to time and there was lessen delay completion time of vessels. 


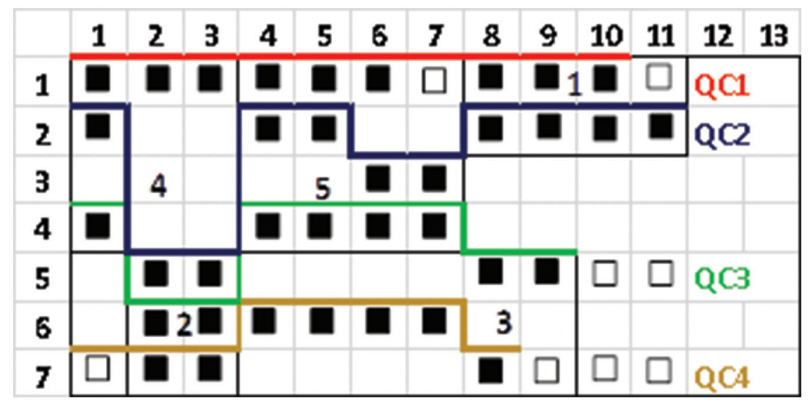

Figure 2 A time space diagram for the exact solution of simultaneous IBAPCQCSP (LVF rule).

Table 5 The exact solution for simultaneous IBAPCQCSP (SPTF rule)

\begin{tabular}{llllll}
\hline$i$ & 1 & 2 & 3 & 4 & 5 \\
\hline$b p_{i}$ & 5 & 1 & 1 & 4 & 1 \\
$b t_{i}$ & 5 & 1 & 3 & 1 & 7 \\
$e_{i}$ & 9 & 3 & 7 & 5 & 12 \\
$r_{i q}^{1}$ & 5 & 1 & 3 & 1 & 7 \\
$r_{i q}^{2}$ & 5 & 1 & 3 & 4 & 7 \\
$r_{i q}^{3}$ & - & 1 & 3 & - & 9 \\
$r_{i q}^{4}$ & - & - & - & 4 & 8 \\
\hline
\end{tabular}

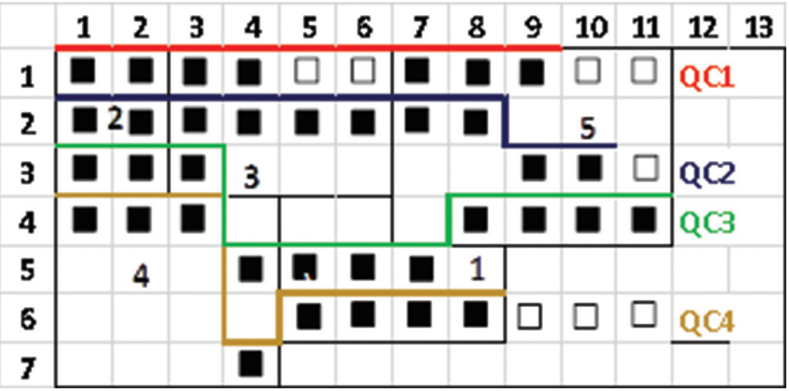

Figure 3 A time space diagram for the exact solution of simultaneous IBAPCQCSP (SPTF rule).

\section{Conclusion}

The highlight of this paper is the integration on operating both resources which are berths and quay cranes at the port terminal simultaneously. There exist two processes namely, BAPC and QCSP which increase the operations efficiency at container terminal. This paper proposed a mathematical 
formulation for the simultaneous IBAPCQCSP with multiple objectives. This study also performed simple numerical experiment to evaluate the performance and validate the simultaneous integrated model with multiple objectives added using LINGO 15.0. For future studies, metaheuristic approach will be considered for the simultaneous integrated model with real life data.

\section{Acknowledgment}

The authors would like to thank the Malaysian Ministry of Higher Education for their financial funding through Fundamental Research Grant Scheme (FRGS) vote number R.J130000.7809.4F470. This support is gratefully acknowledged. The author would also like to thank UTM Centre for Industrial and Applied Mathematics and Department of Mathematical Sciences, Faculty of Science, UTM for the support in conducting this research work.

\section{References}

[1] Han, X. L., Lu, Z. Q., and Xi, L. F. (2010). A proactive approach for simultaneous berth and quay crane scheduling problem with stochastic arrival and handling time. Eur. J. Operat. Res. 207.3, 1327-1340.

[2] Daganzo, C. F. (1989). The crane scheduling problem. Transport. Res B Methodol. 23.3, 159-175.

[3] Park,Y. M., and Kim, K. H. (2005). "A scheduling method for berth and quay cranes." in Container Terminals and Automated Transport Systems. Berlin: Springer, 159-181.

[4] Zhang, C., Zheng, L., Zhang, Z., Shi, L., and Amstrong A. J. (2010). The allocation of berths and quay cranes by using a sub-gradient optimization technique. Comput Ind. Eng. 58.1, 40-50.

[5] Aykagan, A. (2008). Berth and quay crane scheduling: problems, models and solution methods. ProQuest, Georgia Institute of Technology, Georgia.

[6] Wang, R. D., Cao, J. X., Wang, Y., and Li, X. X. (2014). An integration optimization for berth allocation and quay crane scheduling method based on the genetic and ant colony algorithm. Appl. Mech. Mater. 505-506, 940-944. 


\section{Biographies}

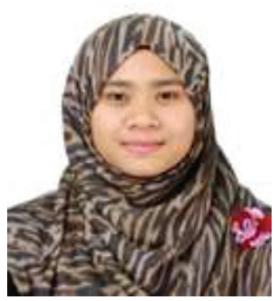

N. Idris is a master student at the Universiti Teknologi Malaysia at Skudai, Johor, Malaysia since 2014. She attended the Universiti Teknologi Malaysia, Johor where she received her B.Sc. in Industrial Mathematics in 2014. Nurhidayu is currently completing a master' degree in Applied Mathematics specialized in Operational Research field at the Universiti Teknologi Malaysia at Skudai, Johor, Malaysia. Her master work discusses on minimizing the operation at port which involving Berth Allocation and Quay Crane Scheduling Problem.

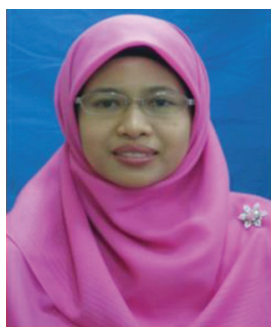

Z. M. Zainuddin is a Senior Lecturer at Universiti Teknologi Malaysia, Malaysia. She received his B.Sc. degree in Mathematics from University of Southampton, United Kingdom in 1996, M.Sc. degree in Operational Research and Applied Statistics, from Salford University, United Kingdom, in 1999, and his Ph.D. degree in Mathematics and Statistics from University of Birmingham, United Kingdom, in 2004. Her research interest includes modeling and solving real life problem using Operational Research, Optimization and Heuristic techniques. She is a member of the OR Society, Management Science/OR Society of Malaysia (MSORSM) and Malaysian Mathematical Sciences Society (PERSAMA). 
